\title{
Metodologias ativas de ensino-aprendizagem para educação farmacêutica: um relato de experiência*
}

\section{Introdução}

Em tempos de sociedades globalizadas e acesso massivo da população à informação por meio de mídias digitais, faz-se necessário o repensar de nossas metodologias de ensino utilizadas diariamente, a fim de se agregar maior conhecimento, tornar o dia a dia da sala de aula mais dinâmico, além de proporcionar a quebra do paradigma professor-aluno, uma vez que a velocidade da construção do conhecimento favorece a complementação destes personagens, que, cada vez mais, aprendem juntos.

Neste contexto, o aluno não deve mais ser visto como um ser passivo, mas estimulado a construir seu conhecimento por meio da avaliação da informação disponível, sendo o professor o responsável pela orientação adequada, pelo acompanhamento e pelo estímulo constante pelo aprendizado de qualidade.

A utilização de recursos de aprendizagem em sala de aula deve contemplar o universo de ferramentas disponibilizadas pela internet e softwares de computador, considerando que o aluno de hoje possui familiaridade com estes recursos, uma vez que grande parte os utiliza em suas atividades diárias de estudo, e, sobretudo, de entretenimento.

Observa-se uma evolução profunda na internet, que surgiu como mera rede de interligação entre computadores, e que hoje representa um espaço interativo do qual todos nós somos parte integrante (Santos, 2010). Pode-se, além de acessar conteúdos nas mais diversas áreas de conhecimento, produzir textos, complementar informações, gerar avaliações, contribuindo para o ensino à distância ou para a complementação das atividades diárias de sala de aula, mediante utilização da internet 3.0.

Acompanhando este processo constante de mudança, a educação de novos profissionais de saúde necessita de reformulações, visando formar profissionais adequados às necessidades de saúde da população brasileira e do Sistema Único de Saúde, integrando a efetiva articulação das políticas de saúde com a educação. Dá-se ênfase à educação problematizadora, centrada no estudante, que constrói seu conhecimento e desenvolve um discurso próprio de maneira ativa através de novas metodologias de ensino e aprendizagem, com o professor no papel de facilitador do processo. Nesta situação, o futuro profissional de saúde é convidado

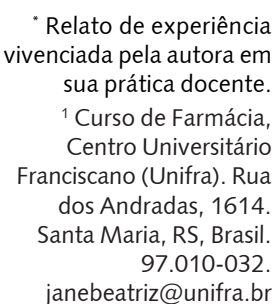

de experiência ua prática docente. Centro Universitário ciscano (Unifra). Rua Santa Maria, RS, Brasil janebeatriz@unifra.br 
a trabalhar com problemas reais, assumindo responsabilidades crescentes e interagindo com a população e os profissionais de saúde das áreas afins.

Este novo profissional exigido pelas últimas reformas curriculares dos cursos da área de saúde, e, em específico, o curso de Farmácia, tem perfil generalista, humanista, crítico e reflexivo, para atuar em todos os níveis de atenção à saúde, com base no rigor científico e intelectual (Brasil, 2002). Este perfil vai ao encontro das necessidades de mudanças profundas no aparelho formador dos profissionais da saúde, porque o atual, individualista e antropocêntrico, não atende mais às necessidades das pessoas nem do processo de trabalho em saúde (Ferreira, Ramos, 2006).

Dentre a variedade de metodologias ativas disponíveis, se faz necessário escolher aquela que melhor se adapta à fase do curso. Assim, simulações, discussões em classe, dramatizações, mapas conceituais e mentais são adequados aos primeiros semestres, enquanto, em etapas intermediárias e finais, o uso de metodologias de problematização, estudos de caso e aprendizagem baseada em projeto trazem melhores resultados para a formação do egresso (Oliveira, 2010).

A disciplina de Assistência e Atenção Farmacêutica tem por finalidade preparar o estudante de farmácia para a atuação efetiva no ciclo da assistência farmacêutica, contribuindo para a melhoria das práticas de gestão de medicamentos no Sistema Único de Saúde - SUS, dentro do que preconiza a política nacional de medicamentos. Além disso, proporciona enfoque na prescrição, dispensação e utilização de medicamentos pelos usuários, visando o uso racional e contribuindo para a detecção e resolução de Problemas Relacionados a Medicamentos (PRMs).

Visando a adequação da disciplina às novas diretrizes curriculares e considerando o perfil do estudante atual, buscou-se utilizar metodologias ativas de ensino-aprendizagem como ferramenta para a construção do conhecimento do aluno, contribuindo, inclusive, para o despertar da prática em educação em saúde, uma vez que os saberes e fazeres situados na perspectiva da interatividade se manifestam quando há utilização expressiva de interfaces de compartilhamento de informações e de colaboração, inclusive, multiprofissional.

\section{Metodologia empregada}

O primeiro passo para a implementação de metodologias ativas no ensino da atenção farmacêutica foi o entendimento de que o polo de ensino, centrado no professor, teria de ser direcionado para o polo da aprendizagem, centrado no aluno. Isto só é possível por meio da substituição da concepção da teoria antecedendo a prática para a articulação teoria/prática; a saída das concepções de saúde como ausência de doença para a saúde enquanto condições de vida.

A experiência de outros cursos foi relevante na organização e sistematização das práticas a serem realizadas. Nessa perspectiva, postulou-se que a disciplina de Assistência e Atenção Farmacêutica, com carga horária semanal de $51 \mathrm{~h}$, seria submetida a um processo contínuo de construção e reconstrução, considerando a relação entre ensino e a prática profissional, prática e teoria, resolução de problemas e interação professor-aluno-comunidade.

Os encontros com os alunos aconteceram semanalmente, de maneira presencial durante o período da aula e de maneira virtual durante todo o semestre. A proposta da metodologia contemplou a divulgação semanal de estudos de caso de pacientes pertencentes aos grupos específicos constantes no programa da disciplina, e a resolução destes pelos acadêmicos, agrupados em duplas, a fim de fomentar a discussão e a observância de diferentes pontos de vista do mesmo caso. Como ambiente de discussão extraclasse, foi planejado o desenvolvimento de um blog, que reuniria, além dos estudos de caso a serem comentados, fontes de referência on-line para subsidiar as discussões.

Semanalmente, foram elaborados estudos de caso (sete durante o semestre), obedecendo aos seguintes critérios: o foco do estudo se concentra na terapia, e não no diagnóstico; o caso deve possibilitar a identificação de um medicamento que seja de escolha, segundo diretrizes de tratamento preestabelecidas; a ênfase no processo de escolha do medicamento, considerando as peculiaridades do paciente; a identificação e resolução de problemas relacionados a medicamentos. 
No planejamento da avaliação dos alunos, considerou-se que esta deveria estar voltada para as competências, traduzidas no desempenho, por meio de um instrumento de acompanhamento de todo o processo ensino-aprendizagem, como forma de garantir o desenvolvimento das competências necessárias à formação do profissional.

\section{Contextualizando o cenário da prática}

A disciplina de Assistência e Atenção Farmacêutica é ministrada no sétimo semestre do curso de Farmácia da Unifra, Santa Maria, RS, e ocorre concomitantemente ao Estágio em Assistência e Atenção Farmacêutica, permitindo uma correlação entre a teoria e a prática do uso racional de medicamentos. $O$ conteúdo referente à atenção farmacêutica é direcionado ao estudo de grupos específicos de pacientes que apresentam características ou problemas de saúde comuns, tais como: diabetes, hipertensão, doenças infectocontagiosas, distúrbios menores (insônia, resfriados, tosse etc.), ou em grupos de pacientes com características semelhantes, como crianças, idosos, mulheres, gestantes, entre outros.

Primeiramente, foi criado um blog (www.atenfar.wordpresss.com) para divulgação do estudo de caso semanal, bem como a inclusão de materiais bibliográficos, textos de interesse, notícias e links que pudessem auxiliar o estudante na busca pelo conhecimento teórico necessário para a discussão do caso. A ferramenta permite que o estudante insira comentários sobre o caso, sempre acompanhada da referência bibliográfica científica consultada. A leitura do comentário inserido por outras duplas pode, e deve, despertar o olhar crítico e promover a avaliação dos comentários, gerando a discussão necessária para a observação de diferentes pontos de vista, ou diferentes referenciais, sobre o mesmo assunto.

$\mathrm{Na}$ elaboração do estudo de caso buscou-se contemplar diferentes aspectos referentes aos problemas de saúde, farmacoterapia proposta e problemas relacionados a medicamentos, considerando as complexidades comuns ao indivíduo em estudo. Assim, os estudantes receberam a tarefa de, semanalmente, visitar o blog, avaliar o estudo de caso proposto e inserir comentários sobre algum aspecto específico do caso. A avaliação completa do caso gerou um relatório, discutido em aula. Nesse momento os estudantes sentavam em círculo, junto à professora, que selecionava: um voluntário para a tarefa de relator, responsável pela compilação dos resultados obtidos pelos diferentes grupos; e um secretário, responsável pela anotação dos resultados do consenso, que seriam posteriormente divulgados no blog na forma de uma súmula do caso clínico.

Ao final da discussão, os aspectos relevantes do conteúdo que não puderam ser encaixados no caso foram complementados pela professora.

\section{A construção dos casos}

O indivíduo é o conjunto de seus Conhecimentos, Habilidades e Atitudes (CHA), ou seja, compreende as competências necessárias para que a pessoa desenvolva suas atribuições e responsabilidades usando a sua criatividade e inovação (Dutra, 2004). Quando o aprendizado ocorre por meio de metodologias ativas, o conhecimento dos estudantes é comparável ao do método tradicional, porém, seu desempenho em relação às suas habilidades e atitudes é superior, reflexo da visão críticoreflexiva proporcionada pelo método. Além disso, observou-se uma satisfação maior por parte dos acadêmicos quando foram solicitados a avaliar o método, por considerarem que a utilização de casos práticos proporciona uma maior relação com a realidade, facilitando a fixação de conteúdos e promovendo o pensamento crítico.

Apesar de o estudo baseado em problemas focar o aprendizado por parte do aluno, o professor (ou instrutor) tem um papel fundamental na descrição do caso a ser estudado, uma vez que este deve contemplar o conteúdo em questão, considerando a complexidade dos sujeitos. A abordagem dos alunos, em um primeiro momento, pode parecer simples, mas, na prática, a mudança de papéis pode não ser tão facilmente concebida: O professor não é mais o condutor, mas companheiro na busca do aprendizado. A aula tradicional, na forma de palestra, é substituída por perguntas. Saber quais perguntas são importantes nem sempre é tarefa fácil. 
Os casos propostos durante os estudos não foram acompanhados por questões formuladas, uma vez que a avaliação dos aspectos mais importantes do caso faz parte do processo de aprendizagem e proporciona a prática da visão integral do sujeito em estudo. Neste contexto, a atuação do farmacêutico deve contemplar: a descrição adequada do caso, o levantamento e a correlação dos problemas de saúde e dos problemas relacionados a medicamentos, e as condutas para a intervenção farmacêutica que visem o uso racional de medicamentos e a melhoria da adesão e da qualidade de vida do paciente.

Um bom cenário clínico para atenção farmacêutica deve deixar claro o diagnóstico do paciente e contemplar a farmacoterapia em uso, de maneira completa, com dados sobre dosagem, posologia e tempo de tratamento. Outras informações que podem ser importantes contemplam: a utilização de terapias alternativas, como uso de plantas medicinais, fitoterápicos e medicamentos homeopáticos; doenças associadas; aspectos socioeconômicos, como escolaridade, faixa etária, renda familiar; adesão ao tratamento medicamentoso ou não medicamentoso, e grau de informação do paciente sobre o medicamento em uso.

Abordar aspectos socioeconômicos é de fundamental interesse para os estudos, uma vez que visam avaliar a relação custo-efetividade do tratamento e, por consequência, a adesão. Pacientes que não têm acesso a medicamentos por outra via que não o Sistema Único de Saúde devem receber, preferencialmente, prescrições de medicamentos constantes na RENAME (Relação Nacional de Medicamentos Essenciais), sob pena da descontinuidade do tratamento.

A avaliação do nível educacional do paciente permite adequar as informações dispensadas a este paciente de acordo com seu grau de compreensão, permitindo que este fique ciente de seus problemas de saúde e da importância da adesão aos esquemas terapêuticos propostos.

Com base no caso, se faz necessária a determinação do objetivo terapêutico para o paciente, ou seja, busca-se: a cura de um problema de saúde, o controle de um quadro, a resolução de um problema relacionado ao medicamento (interações medicamentosas, dose inferior ou superior à adequada, processos alérgicos, efeitos adversos etc.). É importante ressaltar que a determinação do objetivo terapêutico deixa claro qual é o resultado de intervenção esperado, não cultivando falsas expectativas tanto para o paciente quanto para o futuro farmacêutico.

O conteúdo do caso proposto deve refletir a prática diária profissional, seja o seu local de trabalho uma farmácia comercial, um ambulatório, um hospital, um laboratório de análises clínicas ou outro estabelecimento farmacêutico. Por isso, deve-se buscar a inserção de informações tanto farmacológicas quanto clínicas, como resultados de exames laboratoriais, por exemplo. A correlação de dados laboratoriais com o quadro do paciente permite uma avaliação clínica completa e a visão do paciente em todos os seus aspectos.

A inclusão de informações na história clínica do paciente permite que o caso seja dificultado ou facilitado, dependendo dos objetivos propostos para o caso. A inclusão de efeitos adversos pode, por exemplo, evidenciar problemas relacionados a medicamentos comumente encontrados na prática clínica, e possibilitar a busca por formas de resolução deste problema específico. Outro exemplo de modificação consiste na inclusão de uma gravidez, no caso de paciente do sexo feminino, que vai alterar a necessidade e a segurança na utilização de grande número de fármacos.

Na prática clínica, quando utilizamos casos reais para avaliação, é possível encontrar erros de prescrição no que tange à incompletude de informações no receituário, especialmente quanto: à duração de tratamento, utilização de nomes comerciais em prescrições do SUS, sobreprescrição e padrões de prescrição muitas vezes baseados em informações promocionais de propagandistas de medicamentos. A utilização de estratégias de marketing duvidosas favorece o uso de medicamentos sem a necessária avaliação do risco/benefício e resultando, geralmente, em prescrições economicamente inviáveis.

Quando o acadêmico se depara com a grande quantidade de informações disponíveis acerca de fármacos e terapias medicamentosas, é comum se angustiar com o montante de informações necessárias para a prática profissional. Neste contexto, destaca-se o aprender a aprender, e a busca de informações qualificadas para subsidiar a tomada de decisão para a intervenção farmacêutica. 
Estudos randomizados, duplos-cegos ou controlados por placebo são superiores à opinião de um único indivíduo. Outro aspecto diz respeito a estudos financiados pelas indústrias farmacêuticas, gerando conflito de interesses e desqualificando a informação. Assim, mesmo informações oriundas de portais de pesquisa devem ser avaliadas criteriosamente, evitando situações de viés.

É comum se observarem informações conflitantes quando consultadas em fontes diferentes, por exemplo: livros de farmacologia, cartilhas, artigos científicos etc. Nestes casos, é importante aproveitar este momento para fazer a avaliação da fonte de acordo com critérios como: fator de impacto do periódico ou qualidade do livro consultado.

Durante as atividades, a intervenção do professor deve ser restrita a momentos em que a discussão não avança, ou quando o grupo tem dificuldade de entrar em um consenso. Outro caso que exige intervenção diz respeito a estudantes mais calados, por questões de personalidade. Nestes casos, é importante que a inclusão ocorra por meio de questionamentos ou que estes alunos sejam chamados a sumarizar algum aspecto do caso.

\section{Processo avaliativo}

A avaliação deve oportunizar, a professores, alunos e a própria instituição, um momento de reflexão sobre as práticas desenvolvidas e desempenhos alcançados, bem como oportunizar a elaboração de estratégias para o aperfeiçoamento da aprendizagem. Para tanto é necessário: observar, escutar, acompanhar, registrar, discutir, comparar, intervir, mudar e melhorar continuamente (Costa, 2011).

Durante a execução das atividades, buscou-se ampliar os métodos de avaliações, não apenas de maneira somativa, ao final do processo de ensino/ aprendizagem, mas, também, formativa, durante o processo, por meio da observação dos estudantes, das perguntas, das respostas aos questionamentos, da elaboração de sínteses orais e escritas. É importante considerar: a capacidade de reflexão e análise crítica sobre a situação em estudo, a aplicabilidade e pertinência das soluções apresentadas para a resolução do problema. Compuseram a nota final do estudante testes contemplando: o conteúdo, a participação em sala de aula, a elaboração de relatórios orais e escritos, e discussões em ambiente virtual.

\section{Considerações finais}

Atualmente, o mercado de trabalho exige que o profissional de saúde tenha condições não apenas de reproduzir informações recebidas em sala de aula, mas também, e sobretudo, de produzir seu próprio conhecimento ao longo de sua vida profissional, ampliando seu campo de aprendizagem. Buscase a mudança de um paradigma em relação ao estudante e ao processo de ensino/aprendizagem. Observa-se que o aluno tradicional é pouco motivado, imediatista, passivo, muitas vezes dividido entre o trabalho e o emprego.

A utilização de metodologias ativas na disciplina de Assistência e Atenção Farmacêutica permitiu, aos acadêmicos, construírem o próprio caminho, mais seguros de seu potencial, com maior autoestima, autonomia e motivação, uma vez que ampliou a consciência dos estudantes acerca da tolerância, da ambiguidade e da complexidade, e estimula o respeito a opiniões e experiências diversas. Também foi possível observar: o desenvolvimento de uma maior compreensão sobre o tema, maior retenção de conhecimentos, o despertar para a importância da interdisciplinaridade, sempre tendo, por foco, a resolução de um problema do paciente.

Obviamente, as práticas baseadas em problemas exigem momentos de reflexão para a melhoria da didática frente às dificuldades apresentadas, e constante autoavaliação e replanejamento; além de habilidade comunicativa, exercício de liderança, e observação de aspectos referentes à interdisciplinaridade dos conteúdos envolvidos no curso de farmácia e a complexidade dos indivíduos, sujeitos dos casos, exigindo sensibilização, motivação e participação docente, discente e institucional. Não é um caminho fácil, mas, certamente, é recompensador, já que estudar passa a ser um ato desafiador e motivador. 
METODOLOGIAS ATIVAS DE ENSINO-APRENDIZAGEM ...

\section{Referências}

BRASIL. Ministério da Educação. Resolução CNE/CES n 2, de 19 de fevereiro de 2002. Institui Diretrizes Curriculares Nacionais do Curso de Graduação em Farmácia. Brasília: MEC, 2002.

COSTA, E.M.M.B.C. Avaliação da aprendizagem: da teoria à prática. In: CECY, C.; OLIVEIRA, G.A.; COSTA, E.M.M.B.C. (Orgs.). Melhoria da qualidade da Educação Farmacêutica. Brasilia: Associação Brasileira de Ensino Farmacêutico e Bioquímico, 2011. p.111-28.

DUTRA, J.S. Competências: conceitos e instrumentos para gestão de pessoas na empresa moderna. São Paulo: Atlas, 2004.

FERREIRA, H.M.; RAMOS, L.H. Diretrizes curriculares para o ensino da ética na graduação em enfermagem. Acta Paul. Enferm., v.19, n.3, p.328-31, 2006.

OLIVEIRA, G.A. Uso de metodologias ativas em educação superior. In: CECY, C.; OLIVEIRA, G.A.; COSTA, E. Metodologias ativas: aplicações e vivências em educação farmacêutica. Brasília: Associação Brasileira de Ensino Farmacêutico e Bioquímico, 2010. p.11-33.

SANTOS, A.F.M. Plataformas robustas e adaptáveis para a gestão e massificação de conteúdos dinâmicos: utilização em ambientes educacionais contribuindo para o sucesso educativo no ensino secundário em Portugal. 2010. Dissertação (Mestrado) Comércio Eletrónico e Internet, Universidade Aberta, Lisboa. 2010. 
As práticas e os projetos pedagógicos dos cursos da área da saúde têm sido alvo de intervenções a fim de se contemplarem as necessidades da sociedade contemporânea. A formação do farmacêutico não foge a esta linha, na qual se destaca a correlação entre teoria e prática, ressaltando a necessidade de uma visão integral do indivíduo e do meio que o cerca. Com base nestas considerações, o presente artigo visa abordar a utilização de metodologias ativas de ensino-aprendizagem na disciplina de Assistência e Atenção Farmacêutica do curso de Farmácia do Centro Universitário Franciscano (UNIFRA), relatando avanços e dificuldades observadas. Foram realizados, semestralmente, sete estudos de caso abordando problemas de saúde, farmacoterapia e problemas relacionados a medicamentos. Como resultados, destaca-se a formação de um aluno capaz de intervir e construir o próprio futuro com responsabilidade e comprometimento com a formação humanística e generalista.

Palavras-chave: Metodologias ativas de ensino-aprendizagem. Problematização. Educação farmacêutica.

\section{Active teaching-learning methodologies for pharmaceutical education: a report on experience}

The pedagogical practices and projects of healthcare courses have been the target of interventions aimed at taking into account the needs of contemporary society. The training for pharmacists is no exception and, in this, the correlation between theory and practice is highlighted, along with the need for a comprehensive view of individuals and the environment that surrounds them. Based on these points, this article aimed to address the use of active teaching-learning methodologies in the Discipline of Pharmaceutical Care and Assistance of the Pharmacy Course at the Franciscan University Center (UNIFRA), reporting on the advances and difficulties observed. Each semester, seven case studies covering health problems, pharmacotherapy and problems relating to medications were conducted. The results highlighted the shaping of students who were capable of intervening in and constructing their own futures with responsibility and commitment towards humanistic and general training.

Keywords: Active teaching methodologies. Problem-based learning. Pharmacy education.

\section{Metodologías activas de enseñanza-aprendizaje en educación farmacéutica: un relato basado en la experiencia}

Las prácticas y los proyectos pedagógicos de los cursos del área de salud han sido objeto de intervenciones con el fin de atender las necesidades de la sociedad actual. La formación del farmacéutico no escapa de esta línea, en la que se destaca la correlación entre teoría y práctica, subrayando la necesidad de una visión integral del individuo y del entorno que lo rodea. Con base en tales consideraciones el presente artículo busca abordar el uso de métodos activos de enseñanza-aprendizaje en la asignatura de Asistencia y Atención Farmacéutica del curso de Farmacia en el Centro Universitario Franciscano (UNIFRA), relatando avances y dificultades observadas. Fueron realizados, semestralmente, siete estudios de caso que abordaban problemas de salud, fármacoterapia y problemas relacionados con medicamentos. Como resultado, se destaca la formación de un alumno capaz de intervenir y construir su propio futuro con responsabilidad y compromiso con una formación humanística y general.

Palabras clave: Metodología activa de enseñanza y aprendizaje. Problematización. Educación farmacéutica. 
